Gerhart Saenger participated in the project from which this report derives. Thanks are due to Patricia Zybert for her work on the diagrams.

42. The study was carried out with the assistance of funds from the National Institute of Health (Grants nos. 3-R01-HD-04454 and 5-R01-HD-06751) and the New York State Department of
Mental Hygiene. The writing of this paper was greatly facilitated by the award of a John Simon Guggenheim Fellowship for $1972-1973$ to one of us Dr. M. Susser.

43. Requests for reprints should be addressed to: Z. Stein, M.A.,

M.D., B.Ch., $600 \mathrm{~W}, 168$ th St., New York, N.Y. 10032 (USA). 44. Accepted for publication September 6, 1974.

Pediat. Res. 9: 76-83 (1975)

Birth weight

famine

gestation

Printed in U.S.A.

\title{
The Dutch Famine, 1944-1945, and the Reproductive Process. II. Interrelations of Caloric Rations and Six Indices at Birth
}

growth nutrition placenta

\author{
New York State Department of Hygiene and Surgeons and School of Public Health, Columbia University, \\ New York, New York, USA
}

ZENA STEIN(12) AND MERVYN SUSSER

\begin{abstract}
$\checkmark$
\end{abstract}
\section{Extract}

Effects of maternal exposure to famine during the prenatal period were compared among six indices of reproduction by the use of $\mathrm{Z}$ scores. Maternal weight was affected soonest, then birthweight and placental weight. Length and head size at birth were affected relatively less and only after exposure to more severe nutritional deprivation. Path models were constructed of the interrelations of caloric rations, length of gestation, maternal weight, and fetal dimensions. Above a threshold value of $1,500 \mathrm{Cal}$ daily average official food rations available to mothers in the third trimester, a model in which the placenta is treated as a fetal dimension (or organ) seemed most appropriate. Below the threshold value, a model in which the placenta is treated as a transmitter of nutrients seemed most appropriate.

\section{Speculation}

With severe nutritional deprivation, a pregnant mother interposes a buffer that protects the fetus until her nutrient reserves are exhausted. Under normal conditions the growth of the placenta is determined by the growth of the fetus. Under conditions of severe nutritional deprivation, the needs of the placenta are supplied first, and the placenta may thus restrict fetal growth.

In this paper we aim to elicit a structure of relations among indices of the reproductive process measured at birth. To do this, we have taken advantage of data collected for a study of the effects of the Dutch famine of 1944-1945 on fetal growth. A description of the circumstances of the famine as well as the context of this study and its detailed results are to be published elsewhere (7).

The average diet of pregnant women at different stages of gestation was treated as the hypothetical causal variable under study. We quantified an average diet from the official food ration. There was no doubt about the logical priority of the diet in relation to the available indices of the reproductive process. The dimensions of the infant, placental weight, and the end point of gestation, however, were necessarily measured at the same time. With these dependent variables, therefore, no design could confer certainty either about their ordering in time or about the directions of change among them. To assist us in the difficult task of judging among many alternatives about time order and direction of change, we took successive steps in the analysis of the sequences among the outcomes of the reproductive process. First, we made a graphic representation to show the changes through time of all of the variables concurrently. These graphs suggested a number of conclusions.

We then turned to multiple regression analysis to test, elaborate, and add to these conclusions. By applying these analytic procedures first to a series of three-variable sets, and then to larger sets of variables, we gained a good sense of the relations among the variables and strengthened our grasp of the logical priorities (Tables 1-3). Our object was to arrive at a tenable model of the direction and strength of the paths between all of the variables for which we had measures. Path analysis was necessarily the last step, for it imposes on investigators the salutary discipline of making explicit at the outset the hypotheses underlying their conception of the relations between variables (7-9).

We first present the data in graphic form, then present the path models. The intervening steps of analysis and inference from which we derived the structure of the variables in our path models are detailed elsewhere (7).

\section{GRAPHIC PRESENTATION}

We first graphed the six variables of the reproductive process for three famine-stricken cities against caloric rations and 
calendar time. Our aim was to relate the time order of changes in outcome to changes in nutrition. The sources of data are three large teaching hospitals. In the graphs we plot the grouped monthly means for each variable. Figure $1 a$ shows the three curves for maternal, placental, and birth weights. Figure $1 b$ shows three additional curves for length of gestation, infant length, and infant head chrcumference. Figure $1 c$ illustrates the synchrony of the curves for birth weight and infant length in direction, and their disparity in magnitude. The units of measurement for each variable have been converted into standard deviations from a norm ( $\mathrm{Z}$ scores) based on values for the period of July 1945 to March 1946 . The changes from the given nurm in each variable are thereby made comparable, since all change is measured on a common scale of standard deviation units.

The pattern of time-related changes in the six outcome variables before, during, and after the famine leads us to a number of interpretations. Some of these inferences are more

Table 1. Indices of reproductive process in raw values ${ }^{1}$

\begin{tabular}{|c|c|c|c|c|c|c|c|c|c|c|c|c|c|c|c|c|c|c|}
\hline \multirow{2}{*}{$\begin{array}{l}\text { Year and } \\
\text { month of } \\
\text { birth }\end{array}$} & \multicolumn{3}{|c|}{ Placental weight, $\mathrm{g}$} & \multicolumn{3}{|c|}{$\begin{array}{l}\text { Head circumference, } \\
\qquad \mathrm{cm}\end{array}$} & \multicolumn{3}{|c|}{ Maternal weight, $\mathrm{kg}$} & \multicolumn{3}{|c|}{ Birth weight, $g$} & \multicolumn{3}{|c|}{$\begin{array}{c}\text { Duration of } \\
\text { gestation, wk }\end{array}$} & \multicolumn{3}{|c|}{ Length at birth, $\mathrm{cm}$} \\
\hline & Mean & SD & $\mathrm{n}$ & Mean & $\mathrm{SD}$ & $\mathrm{n}$ & Mean & SD & $\mathrm{n}$ & Mean & SD & $\mathrm{n}$ & Mean & SD & $\mathrm{n}$ & Mean & SD & $\mathrm{n}$ \\
\hline \multicolumn{19}{|l|}{1944} \\
\hline 8 & 576.2 & 130.9 & 41 & 34.4 & 2.3 & 31 & 58.1 & 5.89 & 37 & $3,225.1$ & 743.8 & 41 & 39.0 & 3.2 & 41 & 49.6 & 4.7 & 41 \\
\hline 9 & 620.8 & 146.6 & 36 & 35.5 & 1.7 & 34 & 60.2 & 5.77 & 32 & $3,377.6$ & 721.3 & 37 & 39.6 & 2.3 & 36 & 50.5 & 3.2 & 37 \\
\hline 12 & 536.7 & 117.4 & 39 & 35.1 & 1.6 & 26 & 56.4 & 5.59 & 31 & $3,092.4$ & 566.0 & 33 & 39.3 & 1.8 & 32 & 49.2 & 2.3 & 32 \\
\hline \multicolumn{19}{|l|}{1945} \\
\hline 1 & 546.7 & 114.5 & 34 & 34.6 & 1.9 & 33 & 56.5 & 8.01 & 32 & $3,099.2$ & 630.9 & 37 & 39.2 & 2.2 & 36 & 48.7 & 3.0 & 37 \\
\hline 2 & 496.7 & 100.8 & 30 & 34.2 & 2.0 & 30 & 55.8 & 5.17 & 34 & $2,931.4$ & 705.9 & 35 & 38.7 & 3.6 & 35 & 38.7 & 3.6 & 35 \\
\hline 3 & 532.4 & 94.5 & 34 & 34.0 & 1.8 & 32 & 56.1 & 7.85 & 35 & $3,093.2$ & 463.6 & 37 & 39.7 & 1.0 & 35 & 49.3 & 2.4 & 36 \\
\hline 4 & 502.9 & 79.8 & 37 & 34.8 & 1.6 & 30 & 57.2 & 7.50 & 37 & $3,065.0$ & 434.8 & 40 & 39.8 & 1.2 & 39 & 49.8 & 2.2 & 39 \\
\hline 10 & 599.8 & 126.9 & 43 & 34.9 & 1.8 & 35 & 61.3 & 6.72 & 40 & $3,296.2$ & 603.6 & 45 & 39.7 & 1.1 & 43 & 49.5 & 3.6 & 44 \\
\hline 11 & 567.6 & 117.9 & 37 & 35.2 & 2.2 & 26 & 62.2 & 7.38 & 38 & $3,281.5$ & 795.6 & 39 & 38.6 & 3.8 & 39 & 48.8 & 4.4 & 39 \\
\hline 12 & 616.2 & 134.1 & 34 & 35.5 & 1.6 & 28 & 61.2 & 6.36 & 26 & $3,222.3$ & 781.1 & 35 & 38.1 & 5.7 & 35 & 49.4 & 3.5 & 34 \\
\hline \multicolumn{19}{|l|}{1946} \\
\hline 1 & 608.3 & 144.9 & 60 & 35.4 & 1.7 & 54 & 62.7 & 8.20 & 61 & $3,331.7$ & 754.7 & 63 & 39.1 & 3.2 & 60 & 49.3 & 3.7 & 63 \\
\hline 2 & 566.8 & 146.6 & 56 & 35.2 & 2.3 & 41 & 61.2 & 7.07 & 58 & $3,146.3$ & 820.9 & 60 & 39.2 & 3.5 & 58 & 48.8 & 4.6 & 60 \\
\hline 3 & 610.7 & 144.5 & 75 & 35.1 & 2.3 & 41 & 63.2 & 6.75 & 68 & $3,374.2$ & 579.1 & 76 & 40.0 & 2.2 & 75 & 49.3 & 5.5 & 73 \\
\hline
\end{tabular}

${ }^{1}$ Mean, standard deviation, and number of cases; Rotterdam maternities, from August 1944 through March 1946.

Table 2. Indices of reproductive process in standardized normal deviates ${ }^{1}$

\begin{tabular}{|c|c|c|c|c|c|c|}
\hline Month of birth & Maternal wt & Birth wt & Length at birth & Head circumference & Length of gestation & Placental Wt \\
\hline 44.08 & -2.891 & -0.526 & 0.399 & -2.646 & 0.109 & -0.613 \\
\hline 44.09 & -1.194 & -0.300 & 0.611 & 0.548 & 0.355 & 1.333 \\
\hline 44.10 & -2.325 & 0.951 & 1.668 & 1.710 & 0.355 & 0.761 \\
\hline 44.11 & -1.598 & 1.076 & 1.034 & -1.194 & 0.355 & -1.682 \\
\hline 44.12 & -4.264 & -2.190 & -0.658 & -0.613 & 0.109 & -2.337 \\
\hline 45.01 & -4.183 & -2.965 & -0.658 & -2.065 & -0.137 & -1.115 \\
\hline 45.02 & -4.749 & -4.166 & -2.138 & -3.226 & -1.121 & -4.082 \\
\hline 45.03 & -4.507 & -3.816 & -1.715 & -3.807 & -0.383 & -2.524 \\
\hline 45.04 & -3.618 & -4.054 & -1.927 & -1.484 & 0.109 & -3.811 \\
\hline 45.05 & -4.668 & -3.704 & -1.081 & -0.613 & 0.355 & -2.812 \\
\hline 45.06 & -1.921 & -3.829 & -1.504 & -2.936 & -.875 & -1.621 \\
\hline 45.07 & -0.952 & -0.538 & -0.023 & -1.194 & 0.847 & -0.892 \\
\hline 45.08 & -1.679 & 0.701 & 0.822 & 1.129 & 0.847 & -1.224 \\
\hline 45.09 & 1.230 & 1.689 & 1.880 & 1.710 & -1.137 & 0.901 \\
\hline 45.10 & -0.305 & 1.114 & 0.399 & -1.194 & 0.601 & 0.416 \\
\hline 45.11 & 0.422 & -0.601 & -1.292 & -0.323 & -1.121 & -0.988 \\
\hline 45.12 & -0.386 & -0.188 & 0.188 & 0.548 & -1.367 & 1.132 \\
\hline 46.01 & 0.826 & -1.414 & -1.292 & 0.258 & -1.121 & 0.787 \\
\hline 46.02 & -0.386 & -0.851 & -0.446 & -0.323 & 0.109 & -1.023 \\
\hline 46.03 & 1.230 & 0.088 & -0.235 & -0.613 & 1.339 & 0.892 \\
\hline
\end{tabular}

${ }^{1}$ Mean monthly values in $\mathrm{Z}$ scores of postpartum maternal weight, birth weight, placental weight, length at birth, head circumference at birth, and length of gestation, using as the norm the monthly scores of the postwar period July 1, 1945 to March 31, 1946, Rotterdam, Amsterdam, and Leiden. 
Table 3. Descriptive statistics and correlations for path models

Birth wt, g Maternal wt, kg Fetal age, wk Length, $\mathrm{cm}$ Head circumference, $\mathrm{cm}$ Placental wt, g Calories

\begin{tabular}{|c|c|c|c|c|c|c|c|}
\hline $\begin{array}{c}\text { Famine condition }(<1,500 \\
\text { Cal in } 3 \text { rd trimester })\end{array}$ & & 0.31 & $0.67^{1}$ & $0.78^{1}$ & $0.58^{1}$ & $0.62^{1}$ & $0.22^{1}$ \\
\hline Maternal weignı & & & $0.13^{2}$ & $0.22^{1}$ & $0.22^{1}$ & $0.19^{1}$ & $0.18^{1}$ \\
\hline Fetal age & & & & $0.77^{1}$ & $0.26^{1}$ & $0.36^{1}$ & 0.02 \\
\hline Length & & & & & $0.39^{1}$ & $0.49^{1}$ & $0.11^{3}$ \\
\hline Head circumference & & & & & & $0.30^{1}$ & $0.21^{1}$ \\
\hline Placental weight & & & & & & & $0.25^{1}$ \\
\hline Mean & 3,183 & 57.8 & 39.5 & 49.6 & 34.8 & 553 & 1,040 \\
\hline SD & 595 & 6.9 & 2.2 & 3.3 & 1.8 & 120 & 338 \\
\hline $\mathrm{n}$ & 402 & 367 & 393 & 395 & 345 & 377 & 404 \\
\hline \multicolumn{8}{|c|}{$\begin{array}{l}\text { Nonfamine condition }(>1,500 \\
\quad \text { Cal in } 3 \text { rd trimester })\end{array}$} \\
\hline Birth weight & & $0.19^{1}$ & $0.70^{1}$ & $0.83^{1}$ & $0.54^{1}$ & $0.61^{1}$ & 0.02 \\
\hline Maternal weight & & & 0.03 & $0.10^{3}$ & $0.12^{3}$ & $0.15^{2}$ & $0.15^{2}$ \\
\hline Fetal age & & & & $0.77^{1}$ & $0.25^{1}$ & $0.43^{1}$ & 0.08 \\
\hline Length & & & & & $0.43^{1}$ & $0.47^{1}$ & -0.01 \\
\hline Head circumference & & & & & & $0.24^{1}$ & 0.04 \\
\hline Placental weight & & & & & & & 0.05 \\
\hline Mean & 3,306 & 61.6 & 39.2 & 49.5 & 35.2 & 592 & 2,212 \\
\hline $\mathrm{SD}$ & 703 & 7.3 & 3.4 & 3.8 & 2.0 & 137 & 404 \\
\hline $\mathrm{n}$ & 404 & 381 & 398 & 399 & 298 & 398 & 408 \\
\hline
\end{tabular}

$$
\begin{aligned}
& { }^{1} P<0.001 . \\
& { }^{2} P<0.01 . \\
& { }^{3} P<0.05
\end{aligned}
$$

secure than others. All must be seen in the context of our conclusions from the separate analysis of each outcome. Thus we know that nutritional deprivation caused intrauterine growth retardation with little change in length of gestation: and that all the notable effects of the famine on maternal weight and intrauterine fetal growth related to exposure during the third trimester of pregnancy. Furthermore, the effects of nutritional deprivation on birth weight, placental weight, head size, and infant length at birth were not linear like those on maternal weight, but virtually confined to the famine condition. Our graphic analysis (Fig. 1, $a, b, c$ ) suggests the following.

\section{THRESHOLD VALUE OF MATERNAL NUTRIENT INTA KE}

There is a threshold value of maternal nutrient intake around which the strength of nutritional effects change. Above the threshold value, the growth of the products of conception is to a large degree buffered from nutritional deprivation, presumably by maternal food stores. Below the threshold, the distribution of nutrients between mother and products of conception changes; maternal needs gain more priority than they have above the threshold. This is to the detriment of fetal and placental growth but to the advantage of maternal survival.

The level of nutritional deprivation imposed by war time rations before the famine affected maternal weight primarily, and in our data had no noticeable effect on placenta and fetus (10). Only when famine supervened did a sharp fall in placental and birth weights follow the further fall in maternal weight. Maternal weight loss reached a maximum with the sharp fall in the second month of famine, and there was no further loss although the famine continued to be severe. By contrast, placental weight and birth weight continued to fall concomitantly with the decline in rations for a further 2 months before reaching their maximum weight loss. Thus, below the famine threshold, there seemed to be a reallocation of nutritional priorities. We surmise that this distribution of nutrients continued into the early phase of recovery, for in the recovery phase maternal weight rose, if not more sharply than placental weight, distinctly more sharply than birth weight did.

\section{METABOLIC NEEDS OF PLACENTA AND FETUS}

As between placenta and fetus, the metabolic needs of the placenta seem to have priority for circulating nutrients.

The famine retarded the growth in weight of fetus and placenta almost simultaneously, but the fall in birth weight lagged a little behind the erratic course of placental weight in timing and degree. On recovery from the nadir of the last famine months fetal growth lagged a full month behind placental growth. This lag in fetal weight, modest during decline and more marked during recovery, might indicate the presence of a placental barrier in the direct line of supply of nutrients from mother to fetus. The placenta is bathed in maternal blood, and its needs can be met before the nutrients are transmitted to the fetus (2).

\section{BONE GROWTH LESS SENSITIVE TO NUTRITIONAL DEPRIVATION}

The growth of the bones that determine fetal length was less sensitive to nutritional deprivation than the growth of the other tissues, such as fat and muscle, that determine fetal weight. The threshold value for nutrient intake above which growth in length is buffered, however, is probably the same as for fetal weight.

The changes in infant length at birth related to caloric rations in the third trimester were less marked but synchronous with those for birth weight. A significant reduction in infant length associated with exposure in the third timester did not appear until the famine reached its height in February 1945. Thus, most birth cohorts, in accord with the usual "small-for-dates" syndrome, had lost weight while maintaining length. Infants were significantly shorter as well as lighter only after severe and prolonged maternal food deprivation. These 
results are consistent with those of autopsy studies in intrauterine growth retardation (3).

An alternative to the hypothesis of the lesser sensitivity of length to nutrient supply might be that length was affected only by the interaction of famine and another factor such as temperature. The maximum effect on length occurred among births in the two coldest months, and fluctuations in the control areas were not entirely dissimilar. The synchrony of length changes with birth weight changes, however, is against this hypothesis, since birth weight changes did not seem to require the interaction of both factors. A third alternative, that length at birth was affected by exposure in the second trimester of pregnancy as well as the first, can be ruled out by the absence of detectable second trimester effects; there was no deferral of recovery in length at birth after the famine. The statistical analysis of changes in infant length with changes in caloric rations to be reported elsewhere fully support the interpretation of a third trimester effect alone (7).

\section{HEAD SIZE ALSO SENSITIVE TO} NUTRITIONAL DEPRIVATION

The changes in head size on exposure to famine in the third trimester, while more erratic than changes in length, exceeded
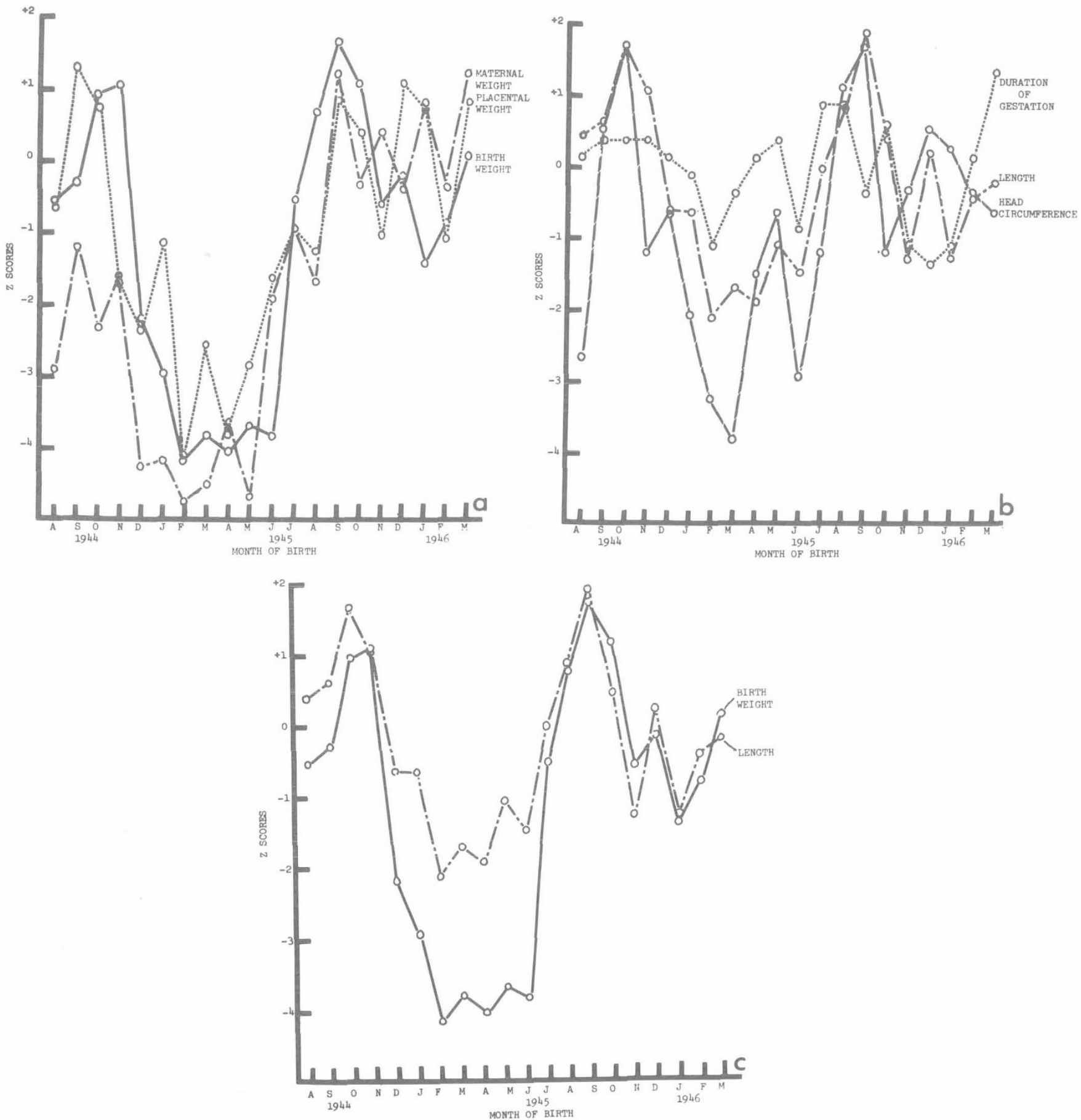

Fig. 1. a: birth weight, maternal weight, and placental weight in the famine cities for the months August 1944 through March 1946 expressed as $\mathrm{Z}$ scores based on the average value of the postfamine period (July 1945 through March 1946). $b$ :duration of gestation, length, and head circumference at birth for the months August 1944 through March 1946, expressed as Z scores based on the average values of the postfamine period (July 1945 through March 1946). $c$ : birth weight and length at birth in the famine cities for the period August 1944 through March 1946 , expressed as Z scores based on the average value of the postfamine period (July 1945 through March 1946). 
them in magnitude. The erratic behavior of the head size curve makes determination of a threshold value difficult, although there is no doubt that it exists as for the other fetal dimensions, and may be higher. If brain growth is a determinant of skull growth, a higher threshold is in accord with autopsy studies which suggest that with intrauterine growth retardation the brain is spared, in that it is among the organs least diminished in size $(3-5)$.

\section{EFFECTS OF FAMINE ON FETAL GROWTH NEITHER MEDIATED NOR CONFOUNDED THROUGH SHORTENING OF GESTATION}

We concluded in the accompanying paper that nutritional effects on length of gestation were equivocal; at most they were small. Simple inspection of the graphs is sufficient to show that reductions in the mean period of gestation could not have accounted for the main changes in other reproductive indices during the famine.

\section{MULTIPLE REGRESSION AND PATH ANALYSIS}

As we have noted, path analysis requires a model in which the position of variables in relation to each other, and the direction of changes between them, must be explicitly assumed and stated. The literature on human reproduction is ambiguous about the direction of change among some of the seven variables included in our analysis. In particular, doubts exist about the relations between caloric intake, maternal weight, and fetal weight, and about the relations between placental and fetal growth. The circumstances of the famine permit us to treat caloric ration as an antecedent and determinant of changes in maternal weight and fetal weight. By inferential procedures, moreover, we have come to the conclusion that under normal nutritional conditions, fetal size is a determinant of placental size; under famine conditions, however, placental size is probably a determinant of fetal size (7). Duration of gestation is assumed to be a measure of fetal age and an antecedent of fetal growth (although strictly it is a measure only of fetal age at birth). Caloric intake, the exogenous antecedent variable in the path model, is a quantified index of caloric rations in the third trimester. Sex has been omitted, since we found no systematic differences in the effects of the famine on males and females. Models have been constructed from the Rotterdam population defined under each of two conditions: (1) the population exposed to the nonfamine condition in which daily rations averaged 1,500 $\mathrm{Cal}$, or more during the third trimester of pregnancy; (2) the population exposed to the famine condition in which daily rations averaged less than 1,500 Cal during the third trimester of pregnancy.

Each condition has been analyzed with two different structures of paths among the variables: Model A, with dimensions of the fetus antecedent to placental weight; Model $\mathrm{B}$, with placental weight antecedent to the dimensions of the fetus.

Our efforts to derive a model in which fetal dimensions and placental weight are in a reciprocal relation failed, and we have no alternative but to present two separate models. On the basis of the analyses leading up to this point, we think it likely that Model A is the more appropriate to the nonfamine condition, Model B to the famine condition.

The path models are so constructed that the variables are placed in their logical order with regard to time and direction, beginning with caloric rations. The vectors lead from each successive variable to the array of dependent variables that flow from it. Along each vector in the path diagram the values for the regression coefficients derived from successive multiple

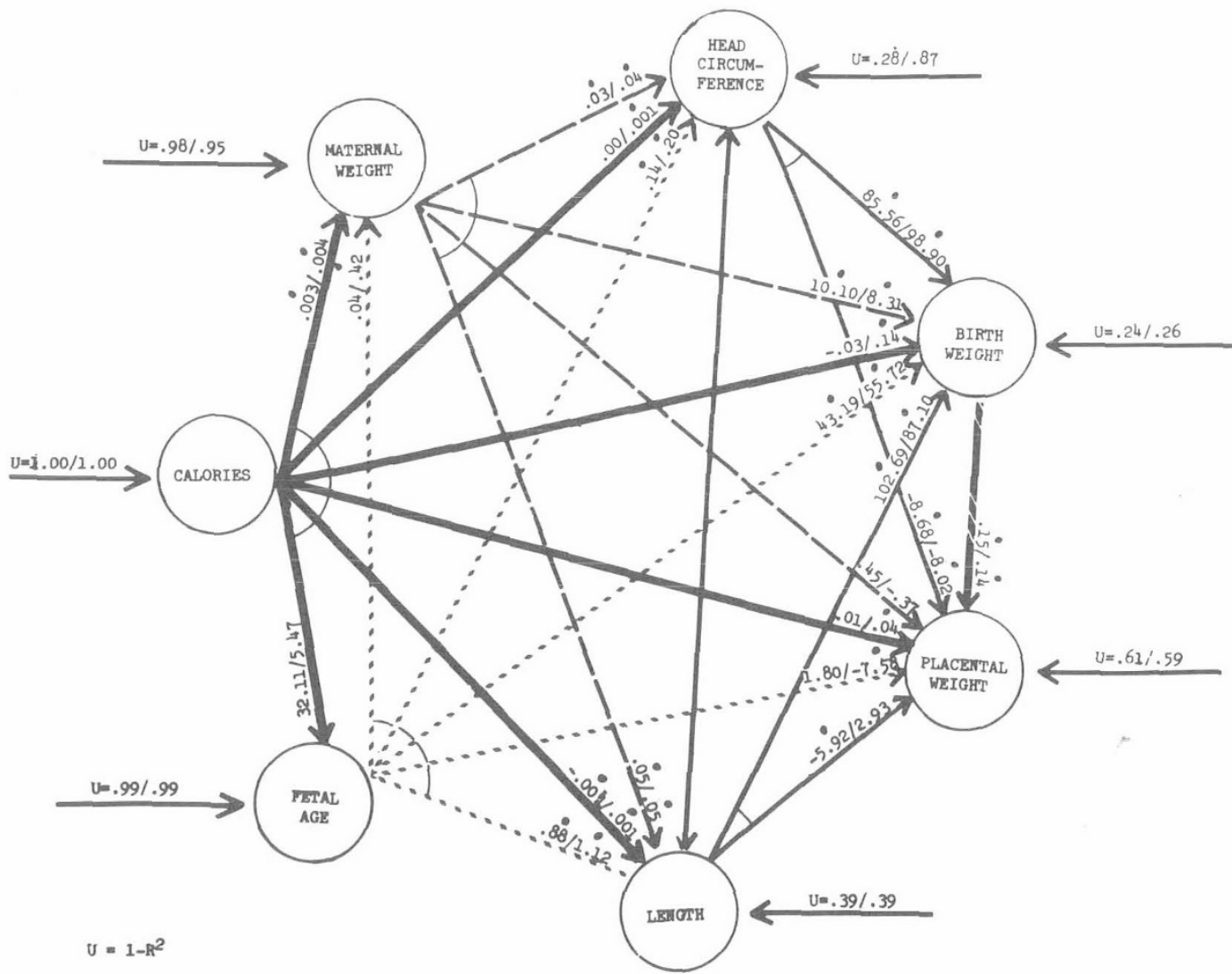

Model A. Path diagram of caloric rations and indices of the reproductive process among individual births in Rotterdam under two nutritional conditions, famine and nonfamine. In this model, fetal growth is assumed to be a determinant of placental growth. Regression coefficients (B values) for each condition are given along the appropriate paths with the nonfamine condition given first. A dot above the B value indicates statistical significance at the $5 \%$ level of probability. U indicates the variance in the variables indicated that remains unexplained by all the antecedent variable included in the model. 


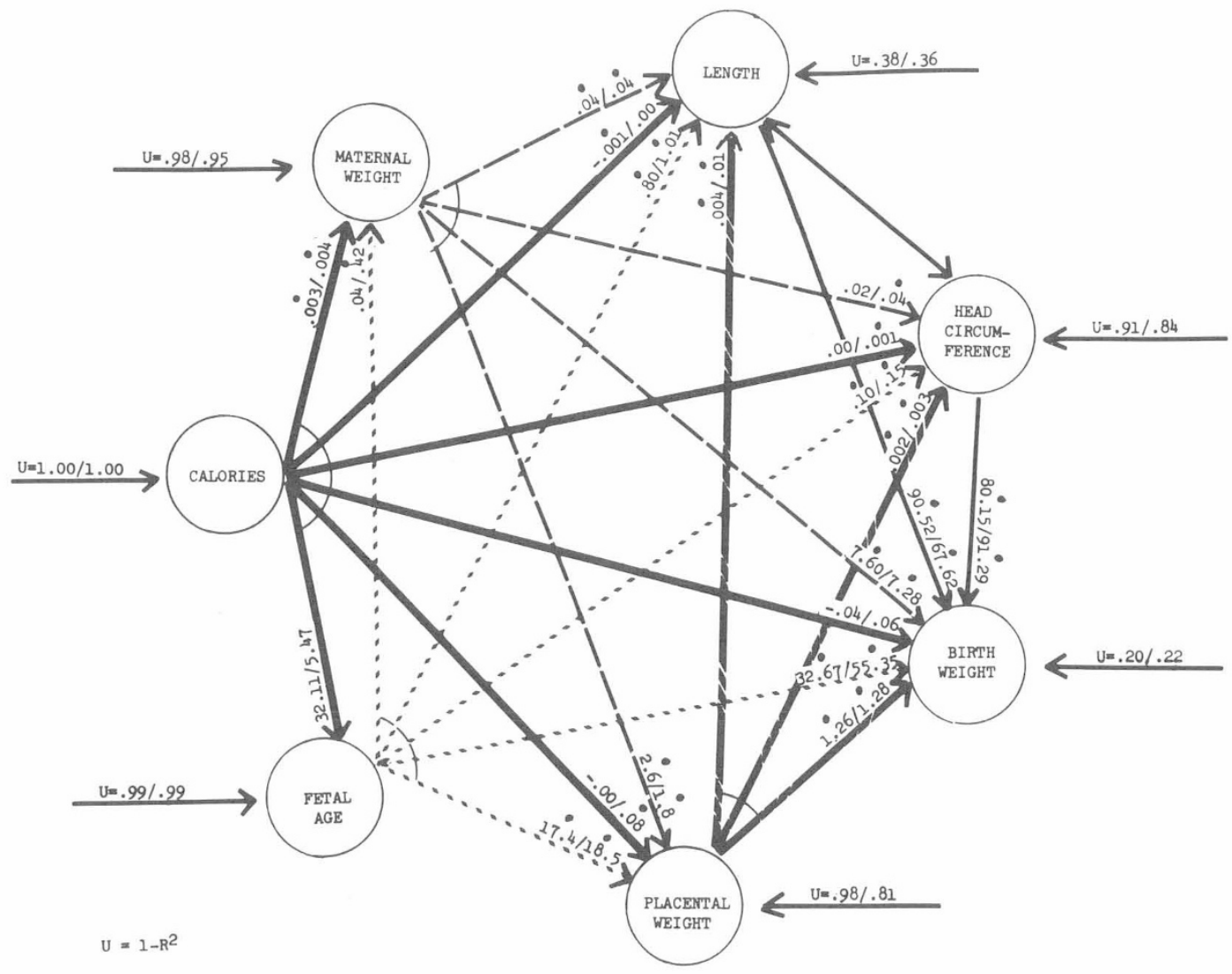

Model B. Path diagram of caloric rations and indices of the reproductive process among individual births in Rotterdam under two nutitional conditions, famine and nonfamine. In this model, placental growth is assumed to be a determinant of fetal growth. Regression coefficients (B values) for each condition are given along the appropriate paths with the nonfamine condition given first. A dot above the B value indicates statistical significance at the $5 \%$ level of probability. U indicates variance in the variable indicated that remains unexplained by all the antecedent variables included in the model.

regression analyses are given. The regression coefficients indicate the amount of change to be expected in the dependent variable to which a particular vector leads, per unit change in the independent variable from which it flows. Two regression coefficients are given for each path, one value for each of the two nutritional conditions, nonfamine and famine

The path models show significant changes between the famine and nonfamine conditions in the magnitude of the coefficients of a number of paths. In both models the changes tend to be in the same direction, although the magnitude of the changes differ. Several changes are in accord with the expectations from the preceding graphic analyses of time changes through the famine.

In brief, both models show that the effect of caloric intake on fetal growth is mediated primarily through other variables, and particularly through maternal weight, and that direct effects of calories on fetal growth are small. Model A, with placental weight as the final outcome, explains about twice as much of the total variance in placental weight $(39 \%$ in the nonfamine condition and $41 \%$ in the famine condition) as Model B with birth weight as the final outcome (which explains $21 \%$ and $20 \%$ of the variance in placental weight, respectively). On the other hand, Model B explains, under the two conditions, respectively, 5 or $6 \%$ more variance than Model A in birth weight, 1 or $4 \%$ more in length, and 1 or $2 \%$ more in head circumference.

\section{INTERPRETATIONS}

In our path models, fetal age is treated as an antecedent condition logically necessary to fetal growth, and can be conceived as an index of the mechanisms intrinsic to the fetus which act together with such extrinsic factors as may affect the rate of growth. The fetal growth process is a determinant of the size of skeletal structures. On logical grounds, skeletal structure in turn is necessarily a determinant of fetal weight. Thus, fetal length and fetal weight can be taken as indices, more or less precise, of the fetal growth process. Since they are determinants of placental weight, the placenta can be considered a fetal organ.

Fetal age is inferred from length of gestation, and otherwise stated marks age at which the transition of birth occurs. By analogy with other developmental processes, chronologic age may be more or less advanced at the time of the transitional event of birth. That accelerated fetal growth may advance the time of birth and reduce age at birth is a limitation on the use of length of gestation as an index of fetal age and hence a determinant of fetal growth; some circularity is involved because fetal growth may thus also determine length of gestation. On the other hand, the insensitivity of length of gestation to the extrinsic factor of nutrition, as well as to such extrinsic factors as social class (1), encourages us to use it as an index of fetal age.

Nutrition is the main exogenous variable in this study, and, like fetal age, it is another necessary condition for fetal growth. The fetal supply is transmitted through the mother and involves her metabolism and her stores. Under normal nutritional conditions, almost the whole of the influence of current caloric intake on fetal weight is in some manner mediated by maternal weight. With the decline of maternal weight under famine conditions, a more direct path of fetal supply that bypasses maternal weight is added, although the mediation of maternal weight in fetal weight continues almost unchanged. We suggest that this mechanism interprets, in our path models, the shift seen in the famine condition towards stronger direct paths between caloric intake in the third 
trimester and birth weight and placental weight. It seems as though in nutritional extremity maternal reserves were exhausted and maternal weight had reached a lower limit. Now a larger share of caloric intake was needed to sustain the mother's metabolic processes. At the same time, the fetus continued to draw on the mother as under normal conditions.

A degree of deficiency in nutrients that failed to sustain normal fetal weight sustained normal growth in length. Where fetal weight is thus diminished without diminution in fetal length, the relative contribution of infant length to the variance in birth weight naturally diminishes, while that of the intrinsic growth process indicated by length of gestation increases.

Fetal growth is a determinant of placental growth. Under normal nutritional conditions, the placenta seems to act to some degree like a passive channel of transmission; it lies like a dam in the direct line of supply of nutrients from mother to fetus. Thus, in the nonfamine condition, nutrient supplies were sufficient to sustain the needs of fetus and placenta for continuing growth as well as for the maintenance of their current metabolic state. The placenta, bathed in the blood of the maternal villi, has first access to nutrient supplies. From these supplies, we postulate, the placenta obtains directly the nutrients to maintain the current metabolic state determined by the development achieved up to that time. The remaining nutrients are transmitted to the fetus, where they are used to maintain the current metabolic state of the fetus, to sustain the further growth of the fetus, and thus in turn to sustain he further growth of the placenta as a fetal organ

In normal nutritional conditions, the amount of nutrients transmitted to the fetus after the placenta has satisfied its current metabolic needs are evidently adequate for the further needs of fetal growth. Hence path models show an indirect pathway from caloric intake through maternal metabolism (indicated by maternal weight) without diversion of caloric intake to the placenta. In famine conditions, however, the nutrients transmitted to the fetus after passage through the placenta were presumably insufficient to sustain normal rates of fetal growth. The appearance of a direct path from caloric intake to placenta then makes evident the placenta's absorption of nutrients and its potential role either as a barrier in the path of the fetal supply, or even as an active agent for the transmission of nutrients.

Some shifts under the famine condition? were not anticipated and have no ready explanation. In Model A, the small negative regression coefficient of the path from infant lengtis at birth to placental weight disappeared with famine. The small regression coefficient betwen fetal age and placental weight became negative with famine in Model A, but not in Model B.

We have been bold in our interpretations of a complex set of statistical interrelations, and we emphasize that at best our path models simplify a manifold reality. They serve as interpretive devices partly because they insure discipline in our constructs of the relations among an array of variables, but mainly because they render some amony the many alternative pathways unlikely, and those that remain, therefore, more likely.

\section{SUMMARY}

Graphic analysis suggested that maternal weight was reduced before the famine, although over the time covered by our data we could detect no obvious effect on the weight of the products of conception caused by this reduction. Maternal weight also absorbed the first impact of the famine. Only after a lower threshold limit of maternal weight was reached did nutritional deprivation seem to affect the products of conception, especially placental weight, by direct paths. On reaching this threshold, mean maternal weight did not continue to fall as the famine progressed. On the other hand, mean placental weight, and then mean birth weight, both of which began to fall later than mean maternal weight, continued to fall and reached their lower limit later than maternal weight. From these circumstances we inferred a reallocation of nutritional priorities between mother and fetus under the famine condition, a reallocation regulated by the placenta. In recovery, the priorities of the nonfamine condition were again restored. With the provision of food after liberation, it seemed likely that the mother and placenta first supplied their own metabolic needs, before the fetus was supplied; maternal weight and placental weight recovered together, but birth weight began to rise. only after a time lag.

Models A and B are in general consisten Under the famine condition, there was a shift in the distribution of caloric rations in the third trimester which brought into play direct paths to birth weight and placental weight. The pathway from caloric intake to maternal weight was strengthened under the famine condition. At this point mothers had priority in the allocation of nutrients, we presume, because they had reached the lower weight limit compatible with survival, and little further weight could be lost. At the same time, the path from maternal weight to birth weight was sustained. Coherent with these relations, the paths between maternal weight and both length and head size at birth were sustained at the same level throughout the famine.

Under the famine condition the paths from infant length at birth to birth weight diminished in strength. These shifts are consistent with the fact that the famine affected length at hirth much less than birth weight; in most affected cohorts, ifants at birth were thin but not short. A compensatory strengthening can be seen in the path from fetal age to birth weight. This presumably reflects a relatively larger contribution of intrinsic growth process to fetal weight as the contribution of fetal length diminished.

\section{REFERENCES AND NOTES}

1. Butler, N. R., and Bonham, D. G.: Perinatal Mortality: The First Report of 1958 British Perinatal Mortality Survey under the Auspices of the National Birthday Trust Fund (E. and S. Livingstone, Edinburgh, 1963).

2. Dawes, G. S.: Foetal and Neonatal Physiology: A Comparative Study of the Changes at Birth (Year Book Medical Publishers, Chicago, 1968).

3. Gruenwald, P.: Amer. J. Obstet. Gynecol., 94: 1112 (1966).

4. Gruenwald, P., and Minh, H. N.: Amer. J. Obstet. Gynecol., 82: 312 (1961).

5. Naeye, R. L.: In: Waisman, H. W. and G. R. Kerr: Fetal Growth and Development, pp. 241-252 (McGraw-Hill, New York, 1970).

6. Smith, C. A.: Amer. J. Obstet. Gynecol., 53: 599 (1947).

7. Stein, Z., Susser, M., Saenger, G., and Marolla, F.: Famine and Human Development: The Dutch Hunger Winter of 1944/45 (Oxford University Press, New York, 1975).

8. Susser, M.: Causal Thinking in the Health Sciences: Concepts and Strategies in Epidemiology (Oxford University Press, New York, 1973).

9. Wright, S.: Ann. Math. Statist., 5: 161 (1934).

10. The data of C. A. Smith (6) collected from clinics in Rotterdam and The Hague suggest that the median levels of birth weight might have been up to $150 \mathrm{~g}$ lower in the wartime prefamine period than in the peacetime postfamine period.

11. We owe much to many willing helpers in The Netherlands and most especially to our Dutch Steering Committee: Professor J. Bastiaans, Chairman; Professor J. Godefroy, Vice-Chairman; Dr. M. J. W. de Groot, Professor C. Den Hartog, Col. J. M. Lagendijk, Professor H. H. van Gelderen, Professor J. J. Groen, Professor N. Speijer, and Mr. A. Straatsma. Dr. F. M. Sorel voluntarily undertook many additional tasks.

Dr. J. J. Huisjes of Groningen, Professor A. Sikkel of Leiden University, Professor G. J. Kloosterman of the University of Amsterdam, Dr. R. J. J. Omer of the St. Elizabeth Clinic in Heerlen and Dr. R. van Wering of the Midwives' Hospital in Rotterdam willingly gave us access to data from their obstetric services. Dr. W. op den Velde, Ms. B. L. Huidekoper, and Ms. H. v. d. Made were also instrumental in gathering obstetric data.

Our colleagues Francis Marolla and Gerhart Saenger participated in the project from which this report derives. Dr. Donald Treiman of the Institute of Policy Research and formerly of Columbia University, guided us through the problems of the path 
analysis. Thanks are due to Patricia Zybert for her work on the diagrams.

The study was carried out with the assistance of funds from the National Institute of Health (Grants nos. 3-R01-HD-04454 and 5-R01-HD-06751) and the New York State Department of

Mental Hygiene. The writing of this paper was greatly facilitated by the award of a John Simon Guggenheim Fellowship for 1972-1973 to M. Susser.

12. Requests for reprints should be addressed to: Z. Stein, M.A., M.B., B.Ch., Columbia University School of Public Health, 600 W. 168 th St., New York, N. Y. 10032 (USA).

13. Accepted for publication September 6, 1974
Collager fibroblasts hydroxyproline
Iysine osteogenesis imperfecta proline

\title{
Dominantly Inherited Osteogenesis Imperfecta in Man: An Examination of Collagen Biosynthesis
}

\author{
GERALD LANCASTER, ${ }^{(35)}$ HY GOLDMAN, CHARLES R. SCRIVER, \\ REYNOLD J. M. GOLD, AND INES WONG \\ deBelle Labcratory for Biochemical Genetics, McGill University-Montreal Children's Hospital \\ Research Institute, Montreal, Quebec, Canada
}

\section{Extract}

We have examined control subjects and patients in an effort to discover a metabolic basis for dominantly inherited osteogenesis imperfecta (OI). Studies were carried out in vitro with cultured skin fibroblasts obtained from OI patients, and in vivo on peptide-bound hydroxyproline excretion in urine. Urinary hydroxyproline excretion (milligrams/24 hr) adjusted for age is essentially normal in OI patients, although the mean excretion rate is below average. The latter finding is presumably a reflection of the smaller body mass of OI patients.

The OI skin fibroblasts, matched for age of donor, site of biopsy, phase of growth, and generation number in culture, incorporate L-proline into hot trichloroacetic acid (TCA)-soluble protein (collagen) at normal rates. The rate of conversion of proline to hydroxyproline in the nascent polypeptide is also normal in OI. Incorporation of L-lysine was also normal in OI. These findings indicate that peptide synthesis of collagen is not impaired in OI.

Rates of galactose incorporation into collagen and the extractability of collagen into normal saline or $0.2 \mathrm{M}$ citric acid were all normal both in OI cells and in the culture medium recovered from the monolayer. These findings, in combination with the urinary data on hydroxyproline excretion in vivo reveal that cross-linking and export of collagen in OI is essentially normal.

The elution profile after ion exchange chromatography of fibroblast collagen on carboxymethyl (CM)-Sephadex was also examined. The normal $2 / 1$ ratio of peak 1 (largely $\alpha 1$ (I) chains) to peak 2 (largely $\alpha 2$ chains) was found in OI fibroblast extracts, which implies that synthesis and initial aggregation of the two types of polypeptide to yield $[\alpha 1(\mathrm{I})]_{2} \alpha 2$ collagen composition is not abnormal in OI.

Despite the negative biochemical findings, a consistent defect in the morphology of OI cells was identified in the log phase and the confluent phase of monolayer cultures. The finding is characterized by irregular packing of the aggregated cells and by an irregular tessellated appearance of the individual OI fibroblast. This observation reassures us that the inherited defect is expressed in vitro.

\section{Speculation}

An abnormality in the primary sequence of polypeptide chain in collagen would be compatible with all of our findings and with the genetics of OI. The mutant allele would affect only about half the products, under the control of only one of the loci determining the polypeptide sequences in collagen chains. Because the OI allele is not expressed in cartilage, a tissue without $\alpha 2$ collagen chains, the defect in OI wnיינה perhaps be found in the $\alpha 2$ polypeptide. However, since the $\alpha 1$ (II) chain of cartilage differs in amino acid composition and in hydroxylysine-linked carbohydrate from the $\alpha 1$ (I) chains of noncartilagenous structures, a defect in $\alpha 1$ (I) chains at the nonhomologous residues will also require investigation.

Osteogenesis imperfecta is a disease characterized by osteoporosis, multiple fractures, and dental involvement (7, 15). Blue sclerae, deafness, and slow healing of wounds are also present to variable extent. Cartilage involvement appears to be minimal or absent. The disease is most commonly inherited as an autosomal dominant with variable expression of phenotype, ranging from the severe congenita form, which is evident in utero and has been diagnosed antenatally (8), to the mild tarda form which generally improves with the onset of puberty.

The dominant mode of inheritance suggests that a structural protein is probably involved in the disease (16). Collagen 